\title{
COMPROBACIÓN DE LOS EFECTOS DE BLOQUEADORES SOLARES COMERCIALES USANDO ENSAYOS BASADOS EN LA SOBREVIVENCIA DE CÉLULAS DE LEVADURA A IRRADIACIÓN UV
}

\author{
Raquel Merino Urteaga ${ }^{* 1,2}$, Sandy Nelly Mansilla García ${ }^{* 1,2}$, \\ Luis Gabriel Gutiérrez Mesías ${ }^{1,2}$, Ana Akemi Kitazono Sugahara ${ }^{1,3^{* *}}$.
}

\begin{abstract}
RESUMEN
Se reportan ensayos basados en la supervivencia de células de la levadura Saccharomyces cerevisiae sometidas a radiación UV, para comparar los niveles de fotoprotección que brindan los bloqueadores solares según su valor de factor de protección solar (FPS). Diez lociones con FPS de 15 a 100 fueron comparadas, usando irradiaciones UVB y UVC. Se observó equivalentes capacidades fotoprotectoras en los bloqueadores con valores de FPS igual y mayor a 50, mientras que los de FPS 15 y 30 mostraron menores niveles de protección que eran proporcionales a esos valores. Asimismo, se compararon los precios por gramo de las lociones analizadas, observándose una gran dispersión. Dado que no se encontraron diferencias notables entre la fotoprotección ejercida por los bloqueadores de FPS $\geq 50$, se puede inferir que esa dispersión es causada por otros factores, como la reputación de la marca, presentación del producto, estrategias de mercadeo y otros. Este trabajo demuestra la necesidad de promover cambios en las normas locales que rigen la venta de los bloqueadores solares, de manera tal que se use la denotación "FPS 50+" para todos aquellos con FPS $\geq 50 \mathrm{Z}$.
\end{abstract}

Palabras clave: Factor de protección solar, FPS, Saccharomyces cerevisiae.

\begin{abstract}
An assay based on the survival rates of UV-irradiated yeast Saccharomyces cerevisiae cells was used to compare the photoprotectant capabilities of several commercial sunscreens with different sun protection factors (SPFs). Ten lotions with SPF values from 15 to 100 were tested using UVB and UVC irradiations. The results showed that all lotions with SPFs $\geq 50$ exhibited similar photoprotectant capabilities. However, the lotions with SPF 15 and SPF 30 exhibited proportionally lower photoprotection levels. Likewise, the prices per gram of the analyzed lotions were compared, finding them widespread. Since the photoprotection levels of the lotions with SPF values above 50 were equivalent, it could be inferred that other

\footnotetext{
* Estas autoras contribuyeron equitativamente a este trabajo - 1 Laboratorio de Química Biológica y Bioanálisis, Universidad Nacional Agraria La Molina - Lima, Perú - 2 Departamento Académico de Biología, Universidad Nacional Agraria La Molina - Lima, Perú - 3 Departamento Académico de Quìmica, Universidad Nacional Agraria La Molina - Lima, Perú

**A Autor de correspondencia, anakitazono@lamolina.edu.pe
} 
factors are responsible for the variations in the prices, such as the recognition of the brand name, product presentation, marketing strategies, and others. This work shows the need to change Peruvian regulations that foresee the selling of sunscreens, to use the notation "SPF $50+$ " for all products with SPF $>50$.

Key Words: Sun protection factor, SPF, Saccharomyces cerevisiae.

\section{INTRODUCCIÓN}

La vida en la Tierra depende de la radiación electromagnética proveniente del Sol, que incluye las del tipo visible, infrarrojo (IR) y ultravioleta (UV). Sin embargo, hace más de 30 años se identificó a la radiación UV como una fuente ambiental altamente dañina para el ADN, que causa mutaciones con potencial efecto cancerígeno ${ }^{1}$. La radiación UV se divide según su longitud de onda en tres segmentos denominados UVA (320-400 nm), UVB (295$320 \mathrm{~nm})$ y UVC (100-295 nm); este último es absorbido por la capa de ozono y por lo tanto no llega a la superficie terrestre. Es por ello que las radiaciones UVA y UVB son las principales responsables de los efectos biológicos de la radiación solar, entre los cuales se incluyen quemaduras solares, pigmentación, envejecimiento prematuro, inmunosupresión y carcinogénesis ${ }^{2}$.

La intensidad de radiación UV, a la cual está expuesta una persona, varía en relación a distintos factores, como la ubicación geográfica. A una mayor altitud la atmósfera es más delgada, por lo que menor cantidad de rayos UV son absorbidos por la capa de ozono en la estratósfera. Según el NIH (National Institutes of Health, EE.UU.) ${ }^{3}$, la exposición a la radiación UV incrementa en un $4 \%$ por cada 300 metros de altura. Por esta razón, las poblaciones asentadas en las zonas altoandinas del territorio peruano se encuentran en mayor situación de riesgo por sobreexposición a la radiación UV. Según el INEI, el $28 \%$ de la superficie peruana la conforma la sierra, considerada como tal a partir de una altitud mínima de $2000 \mathrm{msnm}^{4}$. Así, en un reporte realizado por el Ministerio de Salud en 2013 sobre el análisis de la situación del cáncer en el Perú, se identifica a los departamentos de las zonas altoandinas como los de mayor incidencia de cáncer de piel. Este tipo de cáncer se encuentra entre los cuatro más frecuentes en Arequipa, Ayacucho, Cajamarca, Cusco, Huancavelica y Junín; y entre los ocho más frecuentes en Apurímac, Huánuco, Pasco y Puno 5 .

Las defensas empleadas contra la nociva radiación UV incluyen cubrir la piel con ropa, ampararse en zonas con sombra (uso de sombreros o sombrillas), y usar bloqueadores o protectores solares. Con respecto a estos últimos, desde su aparición en 1928, su uso se ha ido generalizando hasta llegar a ser uno de los recursos más importantes en la prevención al cáncer de piel. La formulación del bloqueador le otorga un determinado nivel de fotoprotección, ya que incluye filtros orgánicos o inorgánicos, los cuales absorben o reflejan la radiación UV incidente ${ }^{1}$. Para los bloqueadores solares, el principal y más aceptado sistema de clasificación es el Factor de Protección Solar (FPS, o SPF en inglés), término acuñado por Greiter en $1974^{6}$. Este método de evaluación numérico relaciona, luego de 16-24 horas, a la dosis eritematosa 
mínima (DEM) de piel protegida con $2 \mathrm{mg} / \mathrm{cm}^{2}$ de un bloqueador, y la de piel expuesta sin protección. El FPS se calcula como la media entre los valores obtenidos al irradiar con UVB a 10 a 20 voluntarios con piel de fototipo I-III, según la clasificación Fitzpatrick ${ }^{1}$. Es también importante notar que, aunque es más práctico relacionar un valor de FPS con los tiempos de exposición requeridos para dar lugar a la formación de un eritema (con o sin bloqueador solar), esta apreciación es errónea. Los valores de FPS están relacionados con la cantidad (o dosis) de radiación UVB, y estas varían notablemente según las características climáticas y la hora del día en las que se da la exposición.

Pese a que la comercialización y el empleo de bloqueadores solares ha crecido a lo largo de los años, la mayoría de usuarios no lo emplean correctamente y muchos aún no lo utilizan. Asimismo, la cantidad de loción aplicada comúnmente por un usuario, que se limita aproximadamente a solo $0,5-1 \mathrm{mg} / \mathrm{cm}^{2}$ de protector, es menor a la cantidad aplicada en las pruebas para el cálculo del FPS $^{7}$. De esta manera, estas variaciones reducen notablemente la efectividad del bloqueador. En nuestro país, algunos estudios realizados en este ámbito han sido dirigidos por la Sociedad Peruana de Dermatología, por ejemplo, en campañas de fotoeducación en playas de Lima y Callao durante el mes de enero del $2012^{8}$. Se evidenció que solo la mitad de la población encuestada utilizaba rutinariamente bloqueador solar. Por otro lado, a pesar de que en un día de playa el $70 \%$ aseguraba usar bloqueador como práctica de fotoprotección, más del $80 \%$ de estos limitaba su aplicación a una sola vez, o a una inadecuada periodicidad (mayor que 2 horas).

Asimismo, los usuarios de bloqueadores solares tienden a pensar que mientras más alto sea el valor de FPS, proporcionalmente mayor será la protección proveída por el producto aplicado. Es importante notar que la "Skin Cancer Foundation" en los Estados Unidos recomienda el uso de bloqueadores solares de amplio espectro (protección contra rayos UVA y UVB) y con valores de FPS mayores que $30^{\circ}$. Además, la FDA (Food and Drug Administration, Estados Unidos) ${ }^{10}$ recomienda el uso de bloqueadores de amplio espectro con FPS de por lo menos 15 o para el caso de personas con piel muy clara, de 30 a 50. Se ha determinado que un bloqueador solar con FPS 15 impide la incidencia del $93 \%$ de los rayos UVB. Este porcentaje se incrementa hasta $97 \%$ para un bloqueador solar de FPS 30 y hasta $98-99 \%$ para uno de FPS 50. Y, dadas las condiciones de aplicación y uso (contacto con agua o sudor, ropa y otros elementos), no es posible lograr un $100 \%$ de protección a partir de un bloqueador solar $^{11}$. En este sentido, desde el año 2007 la normativa que rige la venta de bloqueadores solares en los EE.UU. recomienda que aquellos con valores de FPS mayor a 50 usen la denotación "UVB FPS 50+", para evitar influenciar en los usuarios e impedir que se trate de justificar los altos precios de los mismos ${ }^{12}$.

Para evidenciar de manera didáctica las actividades protectoras de bloqueadores solares de diferentes valores de FPS, es posible usar simples ensayos con la levadura Saccharomyces cerevisiae $^{13}$. En este trabajo reportamos el uso de esta técnica con ligeras modificaciones, para la realización de un análisis comparativo de las actividades fotoprotectoras y de los precios de diez lociones bloqueadoras en un rango de FPS de 15 a 100. 


\section{PARTE EXPERIMENTAL}

\section{Bloqueadores solares y lociones}

Se analizaron un total de diez lociones, las cuales fueron adquiridas en diferentes establecimientos comerciales en la ciudad de Lima (Perú) o donadas por el Laboratorio Skinclean S.R.L. Dos fueron lociones humectantes con adicional protección solar de FPS 15 y 30 (M y E, respectivamente), y las ocho restantes fueron bloqueadores solares con valores de FPS entre 50 y 100 (G, I, J, L, N, P, Q, y R). Los valores de FPS, procedencia y presentación correspondientes están listados en la tabla 1, y las composiciones, en la tabla 2.

Tabla 1. Valores de FPS, procedencia y presentación de las lociones investigadas

\begin{tabular}{cccc}
\hline Loción & FPS & Procedencia & Presentación \\
\hline $\mathrm{M}$ & 15 & Supermercado & Pote de $100 \mathrm{~g}$ \\
$\mathrm{E}$ & 30 & Laboratorio Skinclean & Frasco de $40 \mathrm{~mL}$ \\
$\mathrm{G}$ & 50 & Farmacia & Tubo de $90 \mathrm{~g}$ \\
$\mathrm{I}$ & 100 & Farmacia & Sachet de $10 \mathrm{~g}$ \\
$\mathrm{~J}$ & 90 & Farmacia & Sachet de $10 \mathrm{~g}$ \\
$\mathrm{~L}$ & 50 & Laboratorio Skinclean & Muestra prototipo \\
$\mathrm{N}$ & 100 & Pedido por catálogo & Frasco de $80 \mathrm{~g}$ \\
$\mathrm{P}$ & 50 & Farmacia & Sachet de $8 \mathrm{~g}$ \\
$\mathrm{Q}$ & 60 & Farmacia & Frasco de $150 \mathrm{~mL}$ \\
$\mathrm{R}$ & 50 & Pedido por catálogo & Frasco de $80 \mathrm{~mL}$ \\
\hline
\end{tabular}

Tabla 2. Composición de las lociones investigadas

\begin{tabular}{|c|c|c|c|c|c|c|c|c|c|c|}
\hline Código & $\mathbf{G}$ & I & $\mathbf{E}$ & $\mathbf{R}$ & $\mathbf{P}$ & $\mathbf{N}$ & $\mathbf{M}$ & $\mathbf{J}$ & $\mathbf{Q}$ & $\mathbf{L}$ \\
\hline FPS & 50 & 100 & 30 & 50 & 50 & 100 & 15 & 90 & 60 & 50 \\
\hline \multicolumn{11}{|l|}{ Compuestos fotoactivos } \\
\hline Homosalato & & & & $\mathrm{X}$ & & & & $\mathrm{X}$ & & $\mathrm{X}$ \\
\hline Octocrileno & $\mathrm{X}$ & $\mathrm{X}$ & & $\mathrm{X}$ & $\mathrm{X}$ & & & $\mathrm{X}$ & $\mathrm{X}$ & $\mathrm{X}$ \\
\hline Butil metoxidibenzoilmetano & $\mathrm{X}$ & & $\mathrm{X}$ & $\mathrm{X}$ & & $\mathrm{X}$ & $\mathrm{X}$ & & $\mathrm{X}$ & $\mathrm{X}$ \\
\hline Dióxido de titanio & $\mathrm{X}$ & $\mathrm{X}$ & & & & & $\mathrm{X}$ & $\mathrm{X}$ & $\mathrm{X}$ & $\mathrm{X}$ \\
\hline Dietilamino hidroxibenzoil hexil benzoato & $\mathrm{X}$ & $\mathrm{X}$ & & & $\mathrm{X}$ & & & $\mathrm{X}$ & & \\
\hline Metoxicinamato de etilhexilo & $\mathrm{X}$ & $\mathrm{X}$ & & & $\mathrm{X}$ & & $\mathrm{X}$ & $\mathrm{X}$ & & \\
\hline Octil salicilato & & $\mathrm{X}$ & $\mathrm{X}$ & & $\mathrm{X}$ & & $\mathrm{X}$ & $\mathrm{X}$ & & $\mathrm{X}$ \\
\hline Butilenglicol dicaprilato & & & & & & & & & $\mathrm{X}$ & \\
\hline Ácido fenilbenzimidazol sulfónico & & $\mathrm{X}$ & $\mathrm{X}$ & & & & $\mathrm{X}$ & $\mathrm{X}$ & & \\
\hline Benzofenona 3 & & & $\mathrm{X}$ & & & & $\mathrm{X}$ & & & $\mathrm{X}$ \\
\hline Etil-hexil triazona & $\mathrm{X}$ & $\mathrm{X}$ & & & $\mathrm{X}$ & $\mathrm{X}$ & & $\mathrm{X}$ & & $\mathrm{X}$ \\
\hline Metilen-bis-benzotriazolil tetrametilbutilfenol & $\mathrm{X}$ & & & & & $\mathrm{X}$ & & & & \\
\hline Dietilhexil butamido triazona & & & & & $\mathrm{X}$ & & & $\mathrm{X}$ & & \\
\hline Bis-etilhexiloxifenol metoxifenil triazina & & & & & & & & & $\mathrm{X}$ & \\
\hline 4-metilbencilideno alcanfor & & & & & & $\mathrm{X}$ & & & & \\
\hline
\end{tabular}




\section{Cepas de levadura y condiciones de cultivo}

Las cepas de levadura Saccharomyces cerevisiae utilizadas para todos los ensayos fueron una cepa silvestre y la cepa mutante de deleción rad9 que es altamente sensible a la radiación $\mathrm{UV}^{14}$. Ambas cepas tienen el background W303 con el genotipo: MATa leu2-3,112 trp1-1 can1-100 ura3-1 ade2-1 his3-11,15 . Los cultivos se realizaron en medio YPD (1\% de extracto de levadura, $2 \%$ de peptona, $2 \%$ de glucosa) en líquido, o sólido con $2 \%$ de agar. Las condiciones de cultivo para todos los casos fueron a temperatura ambiente, con agitación rotatoria para cultivos líquidos.

\section{Construcción de la cepa mutante rad9}

En $S$. cerevisiae, el gen $R A D 9$ cumple un rol fundamental en la respuesta al daño al ADN y su eliminación causa un considerable aumento en la sensibilidad a la radiación $U^{14}$. Con el propósito de maximizar la sensibilidad de los ensayos de fotoprotección se procedió a eliminar este gen, usando un método convencional que permite el reemplazo de cualquier fragmento de ADN por un cassette que incluye un marcador de selección. El método usado se basa en el plásmido pUG6 desarrollado por J. Hegemann y colaboradores. Este plásmido incluye el cassette KanMX que confiere resistencia a kanamicina y otros antibióticos ${ }^{16}$. A su vez, el cassette KanMX está flanqueado por sendas secuencias loxP que son reconocidas por la recombinasa Cre lo cual permite, si es requerido, la eventual remoción del cassette de selección y dejar solo una secuencia loxP en el sitio de inserción. Para dirigir el cassette loxPKanMX-loxP al locus RAD9 y permitir su reemplazo, se realizó una amplificación mediante PCR usando como plantilla el plásmido pUG6 e iniciadores con las secuencias:

\section{(5'-TCGTGGATATTTGCAACGATGAGCAATGTGAAGTGAGCAAGATAGCAGCT GAAGCTTCGTACGC-3' y 5'-TCATCTAACCTCAGAAATAGTGTTGTATATATCAT TGTCCGTAATGCATAGGCCACTAGTGGATCTG-3').}

Estos oligonucleótidos incluyen secuencias homólogas al cassettequepermiten suamplificación (secuencias subrayadas), y secuencias que flanquean al gen $R A D 9$ para que el producto de PCR resultante pueda ser correctamente reemplazado mediante recombinación homóloga. Luego de la transformación de la cepa silvestre se seleccionaron los transformantes usando medio de cultivo con el antibiótico G418. Los transformantes fueron analizados después para verificar, por un lado, la aumentada sensibilidad a radiación UV y, por el otro, la eliminación del gen RAD9 y su reemplazo por el cassette loxP-KanMX-loxP. La verificación de la eliminación de RAD9 se realizó mediante PCR, usando el ADN genómico de los transformantes como plantilla y cebadores específicos para RAD9 (5'-TATCGACGAAACCTCAAGGG-3' y 5'-CATCAAACAGCCATGCATGC-3') y para el cassette loxP-KanMX-loxP (5'-GCGTACGAAGCTTCAGCG-3' y 5'-CAGATCCACTAGTGGCCTATGC-3').

\section{Ensayos de fotoprotección in vivo}

Las cepas silvestre y mutante rad9 se cultivaron por tres días en caldo YPD con agitación y a temperatura ambiente. Se retiraron alícuotas de $500 \mu \mathrm{L}$ de los cultivos y se sonicaron brevemente para romper los agregados celulares. Cada suspensión sonicada fue mezclada con agua estéril secuencialmente hasta obtener sendas diluciones de 400, 2000 y 10000 veces. Se sembraron $3 \mu \mathrm{L}$ de cada dilución en dos filas, en secciones de placas YPD y, luego de una 
incubación de 1 hora a temperatura ambiente, se procedió a realizar el ensayo. Paralelamente, cada placa se cubrió con una lámina de celofán dividida en cuatro secciones que contenían las lociones a evaluar (figura 1). Para lograr una distribución homogénea de las lociones se mezcló y esparció cuidadosamente $0,031 \pm 0,001 \mathrm{~g}$ de muestra con $200 \mu \mathrm{L}$ de alcohol $96^{\circ}$. Bajo este método se obtuvieron capas de loción de $2 \mathrm{mg} / \mathrm{cm}^{2}$ en cada cuadrante de la lámina de celofán, para obtener las condiciones recomendadas para la aplicación de bloqueadores en la piel antes de la exposición solar ${ }^{17}$. En algunos de los cuadrantes, se colocó papel de aluminio ("Al”) para proporcionar protección total contra la radiación UV (control positivo), y en otros no se adicionó ningún tipo de protector (“-"”) (control negativo). Finalmente, al distribuir todas las muestras y controles se obtuvieron tres sets, que fueron usados en los ensayos de fotoprotección contra radiación UVB y UVC.

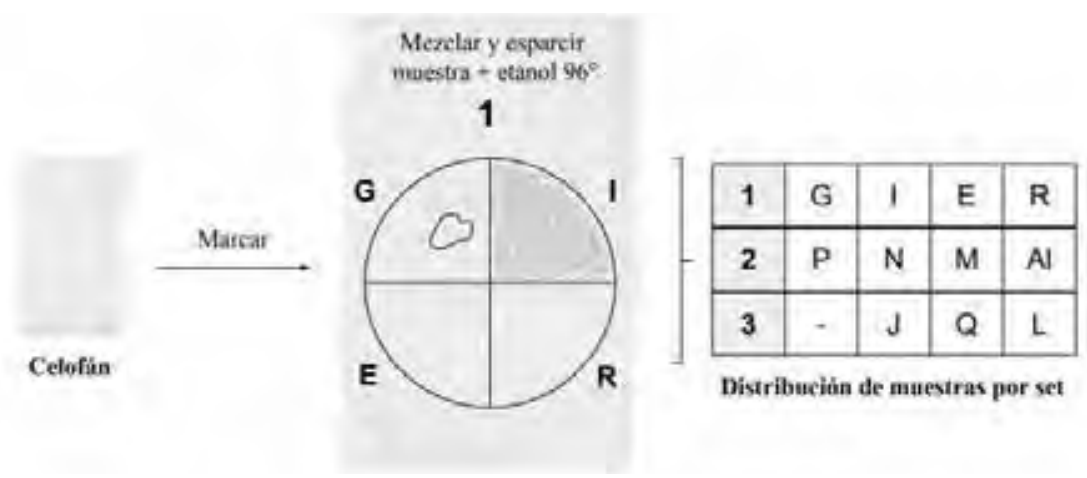

Figura 1. Preparación de las láminas de celofán con las muestras. Se trazaron circunferencias y cuadrantes en cada lámina de celofán, se rotuló con el nombre de cada muestra, control positivo (“Al”) y negativo (“-“). Se agregó 0,031 g de loción y $200 \mu \mathrm{L}$ de etanol $96^{\circ}$ y se homogenizó la mezcla en cada cuadrante para lograr un espesor de $2 \mathrm{mg} / \mathrm{cm}^{2}$. La tabla muestra la distribución de las muestras y controles en cada lámina de celofán, las que fueron usadas para los ensayos de fotoprotección.

Se realizaron ensayos de fotoprotección contra radiaciones UVB y UVC. Para la irradiación UVB (302 nm), la fuente fue un transiluminador VWR (VWR International, EE. UU.) de cuatro tubos de luz de $8 \mathrm{~W}$. El transiluminador se situó $10 \mathrm{~cm}$ por encima de las placas cubiertas con las láminas de celofán. Las placas con cepa silvestre fueron expuestas a radiación UVB a máxima intensidad por 2 minutos y las de la cepa rad9, por 1 minuto 15 segundos.

Para la irradiación UVC (254 nm), las placas cubiertas con las láminas de celofán se colocaron en la base de una cabina de madera que incluía, a una altura de $43 \mathrm{~cm}$, dos lámparas germicidas GE G15T8 (radiación ultravioleta RG-3 de 4,9 W). La exposición para las placas con cepa silvestre fue de 50 segundos y para las placas con la cepa rad9, 25 segundos. Todas las placas fueron incubadas a temperatura ambiente por 1 a 2 días. Los ensayos fueron realizados por duplicado y repetidos por lo menos una vez más, en un día diferente. 


\section{RESULTADOS Y DISCUSIÓN}

Los ensayos de fotoprotección in vivo permitieron un claro análisis comparativo de la actividad fotoprotectora a radiación UV de 10 diferentes lociones. Las figuras mostradas corresponden a resultados obtenidos el mismo día, con un procesamiento simultáneo de las muestras y bajo las mismas condiciones. Se observó que, independientemente de la cepa tratada (silvestre o mutante rad9), prevalecían los patrones de sobrevivencia de cada protector solar, según el tipo de radiación usada (UVC o UVB). En cada ensayo se incluyeron dos secciones control: una desprotegida y totalmente expuesta (“-”), y la otra con total protección por estar cubierta con papel aluminio ("Al"). Estos dos controles establecieron el rango de comparación de las actividades fotoprotectoras.

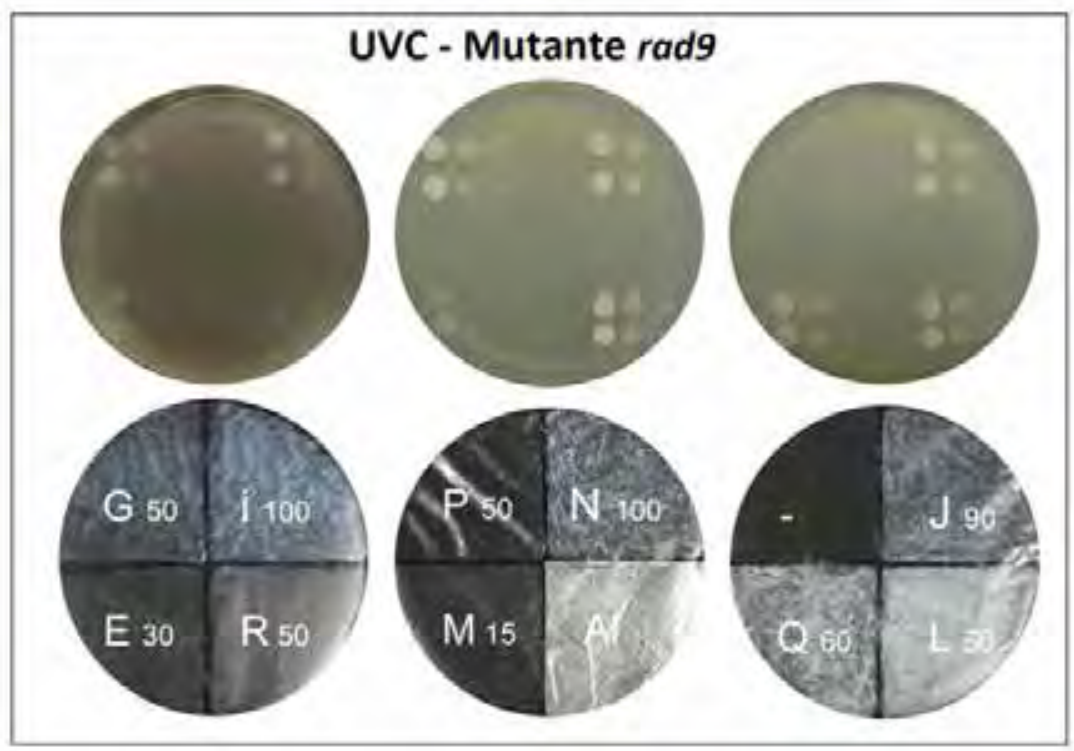

Figura 2. Set representativo de ensayo de fotoprotección a UVC usando la cepa mutante rad9. Se realizaron diluciones sucesivas de 400, 2000 y 10000 veces en cultivos celulares de la cepa. Se plaqueó por duplicado cada dilución formando cuatro sets con dos filas idénticas. Luego de $1 \mathrm{~h}$ cada placa fue cubierta con la correspondiente lámina de celofán previamente preparada. La placa cubierta con la lámina de celofán fue expuesta a 25 segundos de radiación UVC e incubada por 1-2 días, a temperatura ambiente. 
Al utilizar radiación UVC se manifestó una dicotomía en la sobrevivencia de las células de levadura. En base a estos resultados se pudo agrupar a los bloqueadores en dos grupos: aquellos que presentaron un crecimiento abundante y hasta similar al control de protección total, los cuales presentaban un FPS 50+ (FPS de 50-100); y los que por una limitada capacidad de fotoprotección causaron una significativa muerte celular, las lociones de FPS menor a 50. Este comportamiento es reflejado en la figura 2 que muestra resultados representativos de un ensayo de fotoprotección a UVC, usando la cepa mutante rad9.

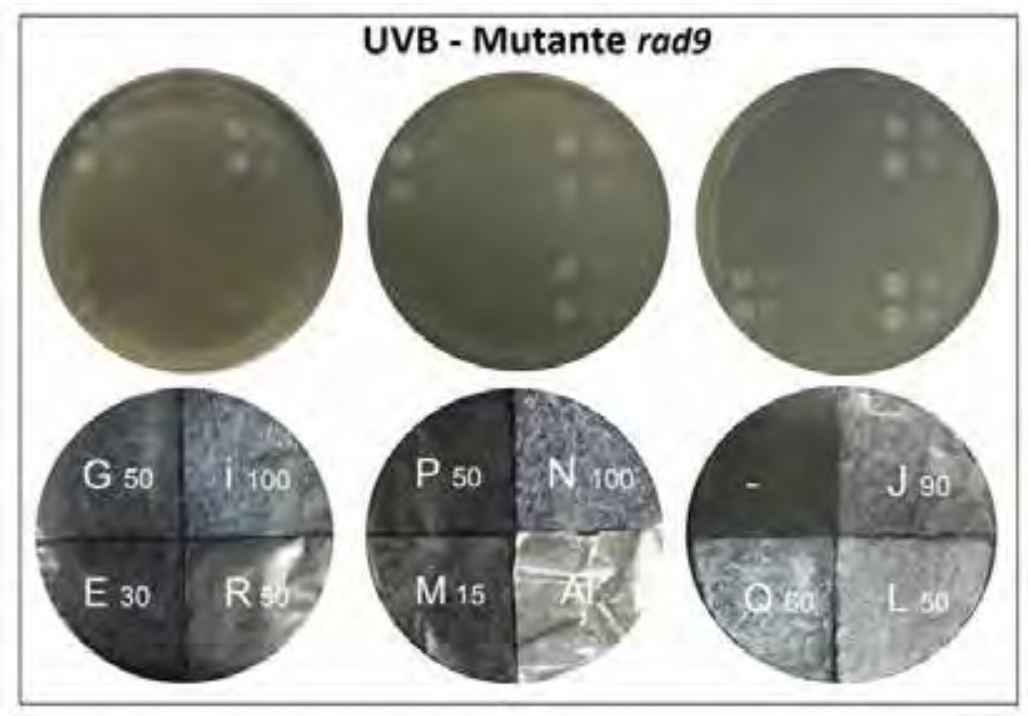

Figura 3. Set representativo de ensayo de fotoprotección a UVB usando la cepa mutante rad9. La preparación de las placas y láminas de celofán fue realizada como se indica en la figura 2. La placa cubierta con la lámina de celofán fue expuesta por 1 minuto 15 segundos a radiación UVB, e incubada por 1-2 días a temperatura ambiente.

En los ensayos de fotoprotección a radiación UVB fue posible una diferenciación más clara entre las lociones pertenecientes al segundo grupo (FPS menor a 50), como se observa en la figura 3. Es así que el crecimiento celular en la sección que contiene la muestra E (FPS 30) presentó un ligero aumento, mientras que la sección con la muestra M (FPS 15) permaneció con escaso crecimiento. Este cambio en la conducta presentada por la loción $\mathrm{E}$ al irradiar con UVB, en comparación al resultado del tratamiento con radiación UVC, se explica por la composición específica que posee, la cual define el correspondiente valor de FPS. Por ello, la presencia solo en esta loción y no en $\mathrm{M}$, de algunos filtros orgánicos con capacidad de absorción a una longitud de onda en el rango UVB, como el octocrileno, podría ser la razón detrás de su aumento en capacidad fotoprotectora. Por otro lado, no se identificaron cambios entre los bloqueadores con FPS mayor que 50, ya que todos permanecieron con similar crecimiento robusto. Sin embargo, cabe destacar que para ambos tratamientos de irradiación (UVC y UVB), la loción R (FPS 50) presentó un exiguo crecimiento, desviándose de los patrones de fotoprotección inherentes a los bloqueadores con tal FPS. 


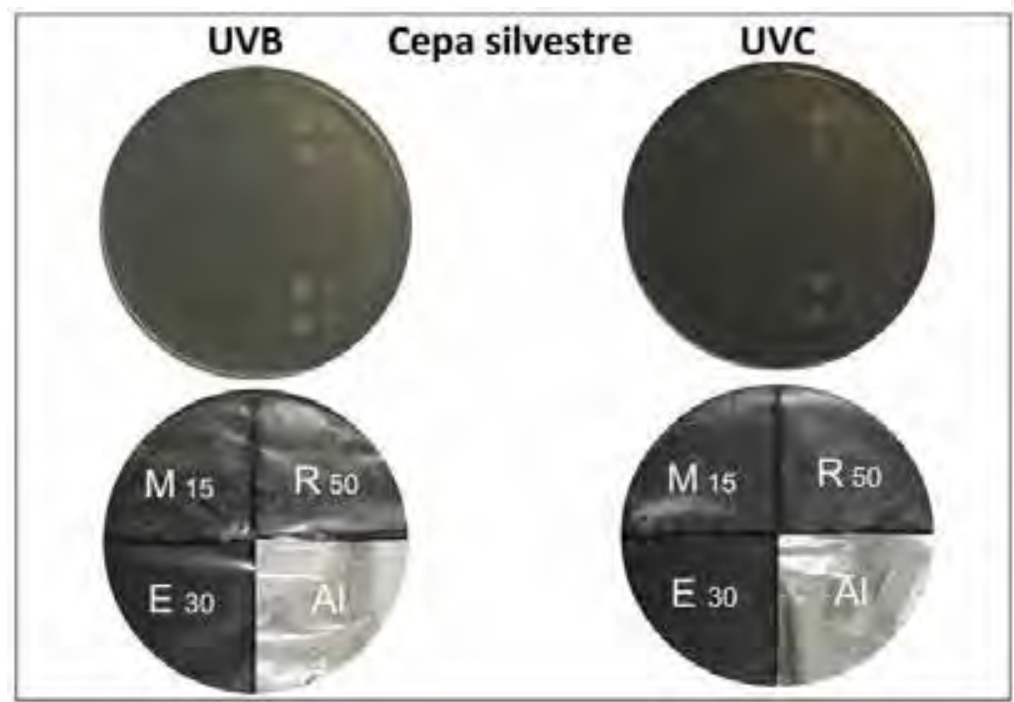

Figura 4. Set representativo de ensayo de fotoprotección a UVB y UVC usando la cepa silvestre sin la incorporación de etanol como diluyente. La preparación del ensayo fue realizada como se indica en la figura 2. La placa cubierta con la lámina de celofán fue expuesta a 2 minutos de radiación UVB o 50 segundos de radiación UVC e incubada por 1-2 días a temperatura ambiente.

Se procedió a analizar este singular comportamiento al realizar pruebas con esta loción sin la incorporación de etanol como diluyente (la única modificación al proceso), y procurando un esparcimiento homogéneo en cada sección. La fotoprotección conferida por R (figura 4) en esta prueba fue notablemente mayor a la previa, llegando a ser comparable a la protección del control con aluminio y significativamente mayor a la otorgada por $\mathrm{M}$, ambas secciones también incluidas en el nuevo ensayo. Se concluyó que en este caso particular la dilución realizada con etanol modifica la distribución de los activos presentes en la loción $\mathrm{R}$, e interfiere con su actividad bloqueadora.

En base a los resultados reportados y bajo las condiciones seguidas en este ensayo es posible afirmar que no fue posible evidenciar diferencias entre las actividades fotoprotectoras de las lociones analizadas de FPS 50 a 100. Por otro lado, la distinción si fue clara para los bloqueadores con FPS menores que 50.

Asimismo, al comparar los precios por gramo de las lociones analizadas (figura 5), se observó una gran dispersión entre los valores. Un caso particular fue el de la loción E, que es comercializada como una loción hidratante que previene el envejecimiento de la piel e incluye además activos fotoprotectores que conducen a un FPS 30, capacidad que fue efectivamente evidenciada en los ensayos. Estas características podrían justificar su alto costo. La dispersión de los valores obtenidos para las demás lociones sí es destacable, considerando que excepto 
por las muestras E y M (FPS 30 y 15), todas presentaban valores de FPS $\geq 50$ (FPS 50+), lo que, según los resultados presentados, implicaba similares capacidades fotoprotectoras. Las muestras comerciales G, P y R tienen valores declarados de FPS 50 y presentan precios por gramo entre 0,177 y 0,488 . Esto implica que para estos productos con similares capacidades declaradas de fotoprotección, un usuario podría estar pagando precios 2,8 veces más altos. Es también importante destacar el alto costo de la muestra $\mathrm{N}$, de una marca muy reconocida en el país, con un valor declarado de FPS 100 y distribuida mediante pedidos por catálogo. Si se considera que las muestras FPS 50+ analizadas presentan similares capacidades fotoprotectoras y se relacionan los precios por gramo $(0,177$ para $\mathrm{G}$ y 0,738 para $\mathrm{N})$, se tendría entonces que un usuario podría estar pagando 4,2 veces más por el mismo efecto fotoprotector.

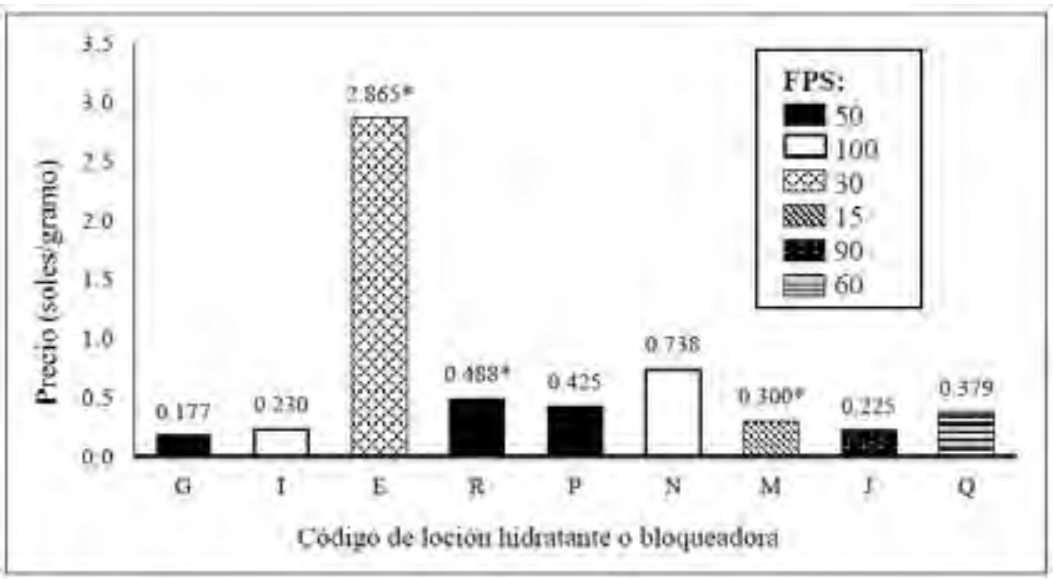

Figura 5. Análisis de los precios por gramo (soles/gramo) de las lociones analizadas. Se calcularon los valores dividiendo los respectivos precios de venta de cada loción entre el peso en gramos del contenido. *Las lociones $\mathrm{E}$ y $\mathrm{M}$ no son comercializadas como bloqueadores solares sino como hidratante y aclaradora, respectivamente. La loción R obtuvo la relación soles/gramo más alta dentro de los bloqueadores de FPS 50.

A pesar de que el sistema FPS es el más aceptado para la evaluación de eficiencia de bloqueadores, la interpretación de éste ha sido por años un tema controversial ya que, al estar basado en respuestas individuales de cada voluntario, no genera una reproducibilidad confiable. Algunas entidades internacionales como la FDA ya proponen que el máximo valor colocado en la etiqueta de lociones sea "50+", decisión respaldada por la falta de evidencia clínica que verifique un beneficio relevante en productos declarados con FPS mayor. Los resultados mostrados corroboran la fútil correlación existente entre estos bloqueadores y sus precios. Es por ello muy importante resaltar que, en lugar de considerar parámetros como marca, alto precio, o valor de FPS, que generan una falsa sensación de seguridad, es más importante tomar en consideración la cantidad del bloqueador usada (obtención de capas de 2 $\mathrm{mg} / \mathrm{cm}^{2}$ ), momento de aplicación (20 minutos antes de la exposición solar), y regularidad en su reaplicación (cada 2 horas o con más frecuencia en casos de excesiva sudoración o baños de agua). 


\section{CONCLUSIONES}

El estudio realizado refleja el margen de correlación existente entre la capacidad de fotoprotección real de una loción bloqueadora según su valor de FPS. Se observa una proporcionalidad hasta un valor de FPS 50. Sin embargo, se demostró que las lociones con valores de FPS mayores que 50 exhiben similar capacidad de fotoprotección. Por esta razón, no se justifica la extensa variación de precios entre lociones bloqueadores con valores de FPS mayores a 50. Es, por tanto, necesario recomendar a los usuarios priorizar el uso periódico y correcto de lociones bloqueadoras de FPS 50+, y evitar ser influenciados por los rótulos que indican valores más altos de FPS, por sus altos precios, o por el reconocimiento de la marca.

\section{AGRADECIMIENTOS}

Nuestro profundo agradecimiento a "InnovatePerú" (Contrato $\mathrm{N}^{\circ} 157-P$ NICP-PIAP-2015) por el financiamiento que hizo posible este trabajo. También agradecemos al Dr. Luis Moromisato (Laboratorio Skinclean S.R.L.) por la provisión de muestras y su valioso apoyo técnico; y a la Srta. Bélgica Pérez, Sr. José Kitazono, e integrantes del Laboratorio de Química Biológica y Bioanálisis en la UNALM por su entusiasta ayuda a lo largo del desarrollo de este trabajo.

\section{REFERENCIAS BIBLIOGRÁFICAS}

1. Schalka S, Reis VM. Sun Protection Factor: Meaning and Controversies. An Bras Dermatol. 2011; 86(3):507-515.

2. Skotarczak K, Osmola-Mańkowska A, Lodyga M, Polańska A, Mazur M, Adamski Z. Photoprotection: Facts and Controversies. Eur Rev Med Pharmacol Sci. 2015; 19(1):98112.

3. Blumthaler M, Ambach W, Ellinger R. Increase in solar UV radiation with altitude. J Photochem Photobiol B:Biol. 1997;39:130-134.

4. Instituto Nacional de Estadística e Informática. Anuario de Estadísticas Ambientales 2013. Lima: INEI; 2014.

5. Dirección General de Epidemiología, Ministerio de Salud. Análisis de la Situación del Cáncer en el Perú. 1ra ed. Lima: DGE/MINSA 2013.

6. Greiter F. Sun protection factor-development methods. Parf Kosm. 1974; 55:70-75.

7. Sivamani, RK, Ghiya M, Maibach HI. Shedding Light on Sunscreens and Their Labels. Am J Prev Med. 2010; 38(6):679-681.

8. Sociedad Peruana de Dermatología. Campañas de prevención de daño solar en bañistas de playas de Lima: educando e investigando para prevenir el cáncer y otros daños en la piel. Lima: SPD 2012.

9. The Skin Cancer Foundation. Sunscreeens explained [Internet] 2012. [Consultado 3 Jun 2018]. Disponible en: https://www.skincancer.org.

10. Food and Drug Administration. CFR - Code for Federal Regulations Tittle 21 [Internet] 2018. [Consultado Diciembre 2018]. Disponible en: https://www.accessdata.fda.gov/ 
scripts/cdrh/cfdocs/cfCFR/CFRSearch.cfm?CFRPart=352\&showFR=1.

11. Sambandan DR., Ratner D. Sunscreens: An overview and update. J Am Acad Dermatol. 2011; 64(4):748-758.

12. U.S. Department of Health and Human Services, Food and Drug Administration (FDA), Center for Drug Evaluation and Research (CDER). Enforcement Policy - OTC Sunscreen Drug Products Marketed Without an Approved Application. Guidance for Industry 2018.

13. Gutierrez L, Romero A, Chávez G, Palomino L, Moromisato L, Kitazono A. Comparison of the photoprotective effects of sunscreens using spectrophotometric measurements or the survivability of yeast cells exposed to UV radiation. Rev Soc Quim Perú. 2017; 83(3):294-307.

14. Paulovich A, Margulies R, Garvik B, Hartwell L. RAD9, RAD17, and RAD24 are required for $\mathrm{S}$ phase regulation in Saccharomyces cerevisiae in response to DNA damage. Genetics. 1997; 145(1):45-62.

15. Thomas BJ, Rothstein R. Elevated recombination rates in transcriptionally active DNA. Cell. 1989; 56(4):619-630.

16. Güldener U, Heck S, Fiedler T, Beinhauer J y Hegemann JH. A new efficient gene disruption cassette for repeated use in budding yeast. Nucleic Acids Res. 1996; 24(13): 2519-2524.

17. Jou PC, Feldman RJ, Tomecki KJ. UV protection and sunscreens: What to tell patients. Cleve Clin J Med. 2012; 79(6):427-436. 